\title{
Implementing cell-free DNA of pancreatic cancer patient-derived organoids for personalized oncology
}

\author{
Zahra Dantes, ${ }^{1}$ Hsi-Yu Yen, ${ }^{2,3}$ Nicole Pfarr, ${ }^{2}$ Christof Winter, ${ }^{4,5,6}$ Katja Steiger, ${ }^{2,3}$ \\ Alexander Muckenhuber, ${ }^{2}$ Alexander Hennig, ${ }^{7}$ Sebastian Lange, ${ }^{1}$ Thomas Engleitner, ${ }^{1}$ \\ Rupert Öllinger, ${ }^{1}$ Roman Maresch, ${ }^{1}$ Felix Orben, ${ }^{1}$ Irina Heid, ${ }^{8}$ Georgios Kaissis, ${ }^{8}$ Kuangyu Shi, ${ }^{9}$ \\ Geoffrey Topping, ${ }^{9}$ Fabian Stögbauer, ${ }^{2}$ Matthias Wirth, ${ }^{10}$ Katja Peschke, ${ }^{1}$ Aristeidis Papargyriou, ${ }^{1}$ \\ Massoud Rezaee-Oghazi, ${ }^{1}$ Karin Feldmann, ${ }^{1}$ Arlett P.G. Schäfer, ${ }^{1}$ Raphela Ranjan, ${ }^{1}$ \\ Clara Lubeseder-Martellato, ${ }^{1}$ Daniel E. Stange, ${ }^{6,7,11}$ Thilo Welsch, ${ }^{6,711}$ Marc Martignoni, ${ }^{12}$ \\ Güralp 0. Ceyhan,, ${ }^{12}$ Helmut Friess, ${ }^{12}$ Alexander Herner, ${ }^{1}$ Lucia Liotta, ${ }^{1}$ Matthias Treiber, ${ }^{1}$ \\ Guido von Figura, ${ }^{1}$ Mohamed Abdelhafez, ${ }^{1}$ Peter Klare, ${ }^{1}$ Christoph Schlag, ${ }^{1}$ Hana Algül, ${ }^{1}$ \\ Jens Siveke, ${ }^{6,13,14}$ Rickmer Braren, ${ }^{5,6,7}$ Gregor Weirich, ${ }^{2}$ Wilko Weichert, ${ }^{2,5,6}$ Dieter Saur, ${ }^{1,5,6}$ \\ Roland Rad, ${ }^{1,5,6}$ Roland M. Schmid, ${ }^{1}$ Günter Schneider, ${ }^{1,5,6}$ and Maximilian Reichert ${ }^{1,5,6}$ \\ ${ }^{1}$ Klinik und Poliklinik für Innere Medizin II, Klinikum rechts der Isar, ${ }^{2}$ Institute of Pathology, ${ }^{3}$ Comparative Experimental \\ Pathology, and ${ }^{4}$ Institute of Clinical Chemistry and Pathobiochemistry, Technical University of Munich, Munich, Germany. \\ ${ }^{5}$ German Cancer Consortium (DKTK), partner site Munich, Germany. ${ }^{6}$ German Cancer Research Center (DKFZ), Heidelberg, \\ Germany. Department of Visceral, Thoracic and Vascular Surgery, University Hospital Carl Gustav Carus, Medical Faculty, \\ Technical University of Dresden, Dresden, Germany. ${ }^{8}$ Institute of Radiology and ${ }^{9}$ Department of Nuclear Medicine, \\ Technical University of Munich, Munich, Germany. ${ }^{10}$ Medical Department, Division of Hematology and Oncology at Campus \\ Benjamin Franklin, Charité, Berlin, Germany. ${ }^{11} \mathrm{DKTK}$, partner site Dresden, Germany. ${ }^{12}$ Department of Surgery, Klinikum \\ rechts der Isar, Technical University of Munich, Munich, Germany. ${ }^{13}$ Institute for Developmental Cancer Therapeutics, West \\ German Cancer Center, University Hospital Essen, Essen, Germany. ${ }^{14}$ Division of Solid Tumor Translational Oncology, DKTK, \\ partner site Essen, Germany.
}

One of the major challenges in using pancreatic cancer patient-derived organoids (PDOs) in precision oncology is the time from biopsy to functional characterization. This is particularly true for endoscopic ultrasound-guided fine-needle aspiration biopsies, typically resulting in specimens with limited tumor cell yield. Here, we tested conditioned media of individual PDOs for cell-free DNA to detect driver mutations already early on during the expansion process to accelerate the genetic characterization of PDOs as well as subsequent functional testing. Importantly, genetic alterations detected in the PDO supernatant, collected as early as $\mathbf{7 2}$ hours after biopsy, recapitulate the mutational profile of the primary tumor, indicating suitability of this approach to subject PDOs to drug testing in a reduced time frame. In addition, we demonstrated that this workflow was practicable, even in patients for whom the amount of tumor material was not sufficient for molecular characterization by established means. Together, our findings demonstrate that generating PDOs from very limited biopsy material permits molecular profiling and drug testing. With our approach, this can be achieved in a rapid and feasible fashion with broad implications in clinical practice.

Conflict of interest: The authors have declared that no conflict of interest exists.

Copyright: (c) 2020, American Society for Clinical Investigation.

Submitted: March 6, 2020

Accepted: June 24, 2020

Published: August 6, 2020.

Reference information: JCI Insight. 2020;5(15):e137809.

https://doi.org/10.1172/jci.

insight.137809.

\section{Introduction}

The identification of molecular targets by genomic profiling has transformed modern oncology, as illustrated by the implementation of precision oncology programs in clinical practice worldwide. However, in selected tumor entities, patients with druggable therapeutic targets clearly remain the minority, particularly in pancreatic ductal adenocarcinoma (PDAC) (1). Currently, PDAC is the third leading cause of cancer mortality in the United States (2) and is projected to be the second cause of cancer death after lung cancer by 2030 (3). Therefore, functionalizing genomic information by patient-derived model systems and 
elucidating vulnerabilities to specific pharmacological perturbation in a clinically adapted time frame are key to overcome these limitations.

Patient-derived organoid (PDO) technology has the potential to meet these requirements and to transform translational cancer research, improving patient outcomes. In recent years, it has become evident that PDOs are able to recapitulate the intra- and intertumor heterogeneity as well as predict response to therapy in the clinic (4-10). However, the use of fine-needle aspiration PDOs (FNA-PDOs) as a resource to establish diagnosis and allow molecular characterization of PDAC patients in a reasonable time frame, even before therapy, remains to be investigated. To perform genotype-guided drug testing, an in-depth genetic characterization of the individual PDO is required. However, the process of PDO expansion to perform subsequent sequencing frequently takes a significant amount of time, limiting the broad application of this technology.

Therefore, we sought to reduce the time required for PDO-augmented molecular diagnosis and functional testing of PDAC patients. To this end, we analyzed the cell-free DNA (cfDNA) in PDO culture-conditioned media (PDO supernatant) as early as 72 hours after biopsy to detect genetic alterations in parallel to the PDO expansion phase to perform pharmacotyping in a clinically relevant model and time frame. Here, we demonstrate that cfDNA in the supernatant of PDOs (PDO-SN) reflect genomic alterations of the primary tumor (PT) and allows immediate genomic characterization and accelerated drug testing of PDOs even from patients with a very limited amount of tumor cell material to facilitate precision medicine in pancreatic cancer.

\section{Results}

Generation of pancreatic cancer PDOs from limited biopsy material. In clinical practice, endoscopic ultrasound-guided FNAs (EUS-FNAs) and consecutive cytological and/or histopathological analysis are widely used to assess pancreatic masses that are suspicious for PDAC. It has been demonstrated previously that PDOs can be generated successfully from both surgical specimens and EUS-FNAs (4, 11-15). Here, we generated $10 \mathrm{PDO}$ lines from individual PDAC patients who either received EUS-FNA (identification [ID] numbers 34, 42, 66, 76, 77, and 121) or surgical resection (ID numbers 25, 48, 54, and 61) (Figure 1A, Supplemental Figure 1, and Supplemental Table 1; supplemental material available online with this article; https://doi.org/10.1172/jci.insight.137809DS1). An additional needle-pass was performed during the procedure for PDO generation from EUS-FNA. To improve the PDO take rate, we have optimized the workflow so that processing of each biopsy is initiated within 30 minutes after biopsy. Successfully established PDOs could be expanded for at least 5 passages and withstood at least 1 freeze-thaw cycle. Interestingly, in some cases (ID numbers 34 and 66) we were able to generate PDOs from biopsies in which a definitive diagnosis of pancreatic cancer by cytological or histopathological means could not be reached (Figure 1A and Supplemental Table 1). However, because an additional needle pass was processed for PDO culture, we cannot exclude that 1 needle pass contained more tumor cells than a previous or subsequent pass from the same pancreatic mass. Similarly, patient ID number 42 displayed 5\% dysplastic cells, and biopsies of patient ID numbers 76 and 77 were considered suspicious by a board-certified pathologist (Figure 1A). These findings indicate the remarkable potential of PDO technology to expand very limited patient biopsy material to perform downstream functional testing. This is particularly meaningful in patients with insufficient biomaterial to perform tissue-driven molecular profiling.

To demonstrate the tumor-initiating capacity of these PDOs, organoids were transplanted orthotopically into athymic nude mice (NU[NCr]-Foxn $\left.1^{n u}\right)$ to generate PDO-derived xenografts (PDOXs) $(n=6)$ (Figure 1B and Supplemental Table 1). Fifty days after transplantation, all transplanted mice showed tumor engraftment (Figure 1, B-D). Hematoxylin and eosin staining of the PDOs generated from FNA specimens $(n=3)$ (Figure 1C) and resection materials (Res.) $(n=3)$ (Figure 1D) showed multifocal duct-like formation with single to multilayered pleomorphic cells, and orthotopic tumor formation could be observed with either solid or cystic morphology (Figure 1, C and D).

Molecular subtypes of matched PTs, PDOs, and PDOXs display remarkable plasticity. Mutations as well as gene expression signatures identified in PDOs have potential implications for the development of novel therapeutic approaches in personalized medicine (4). To further characterize PDOs, corresponding PDOXs, and tumor tissues, we performed PDAC subtyping based on IHC for KRT81 and HNF1A (16, 17) as surrogate markers of PDAC subtypes published by Collisson et al. (ref. 18, Figure 1E, and Supplemental Tables 2 and 3). In the past, molecular subtyping based on gene expression profiling taught us that the complexity of pancreatic cancer can be reduced to allow meaningful patient stratification $(1,18,19)$. 
A

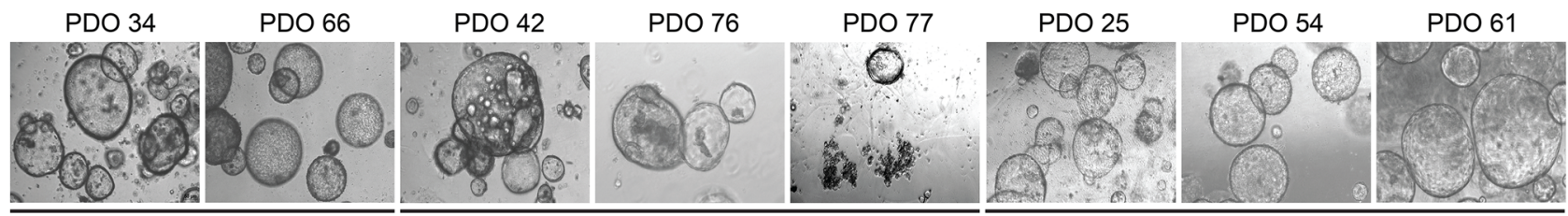

EUS-FNA (neg. cytology)

EUS-FNA (pos. cytology)

Surgical Resection

B

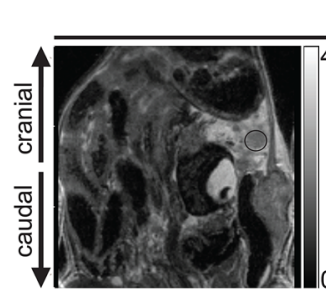

T2w anatomy
PDOX ID 42

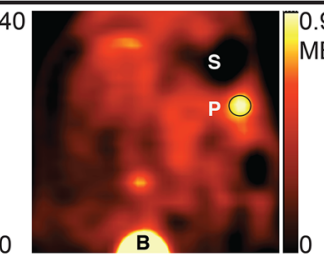

18F-FDG-PET

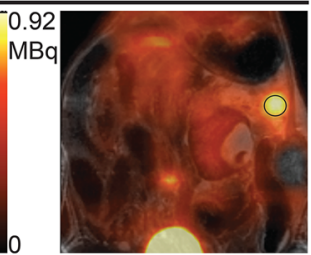

overlay T2w/PET

C
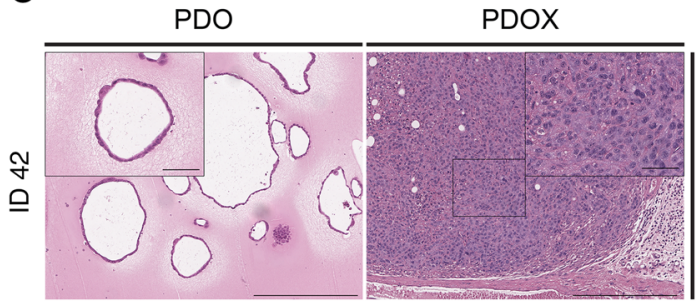

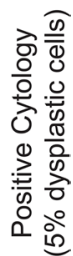

D PDO PDOX
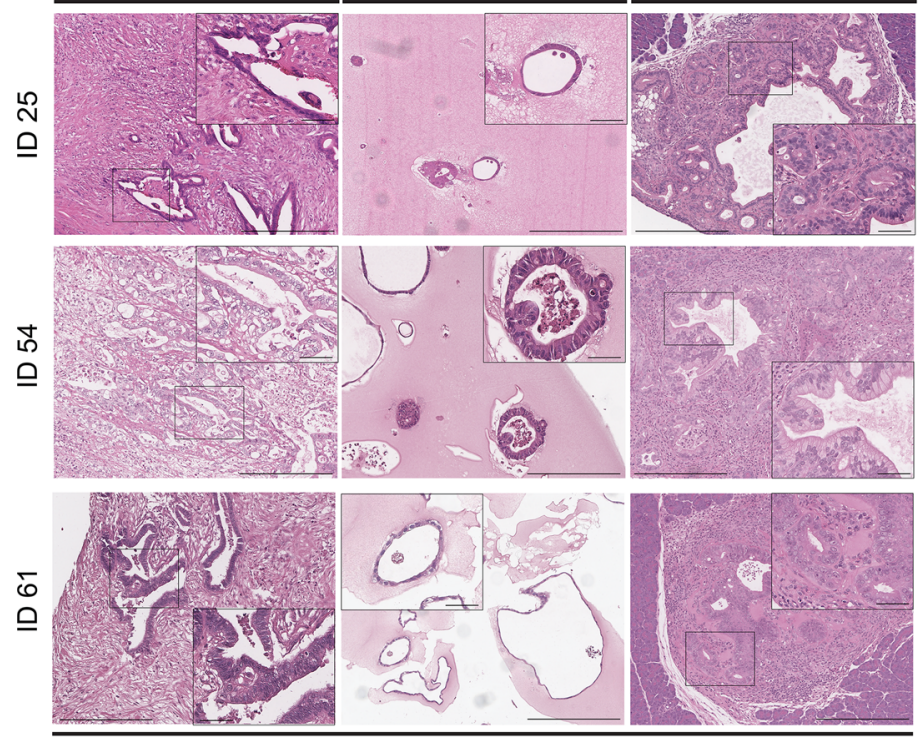

Surgical Resection

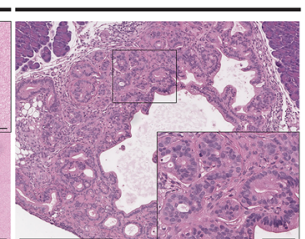

芦
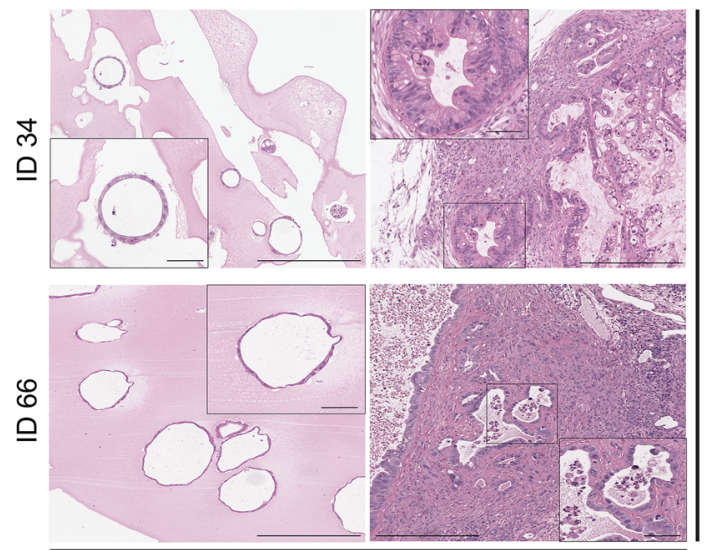

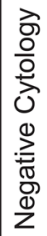

EUS-FNA
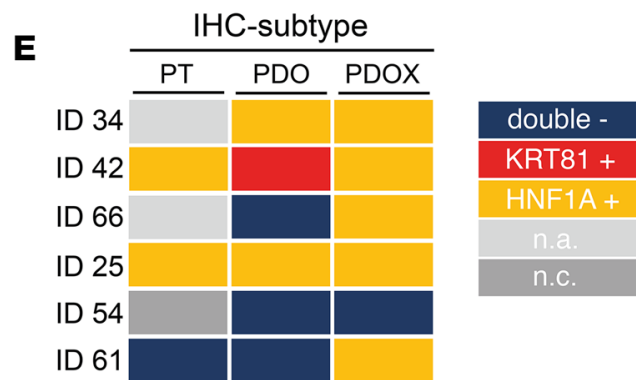

Figure 1. Expansion and characterization of pancreatic cancer PDOs from limited biopsy material. (A) Representative phase-contrast images of cytology-negative ( $n=2$; PDO 34 and 66) (neg. cytology), cytology-positive/suspicious ( $n=3$; PDO patient ID numbers 42, 76, and 77), PDOs (pos. cytology), and PDOs generated from resection specimens ( $n=3$; PDO patient ID numbers 25, 54, and 61). Scale bars: $50 \mu \mathrm{m}$. (B) Representative experiment of PDOX generation ( $n$ = 6). PET/MRI image of PDOX (ID 42) 50 days after orthotopic injection of PDO (patient ID number 42). Left to right: Coronal T2-weighted MRI for anatomic correlation, corresponding PET image demonstrating high focal fluorine-18 (18F) fluorodeoxyglucose (FDC) uptake in the tumor region (blue circle), and fused image (stomach [S], pancreas [P], and bladder [B]). Arrows indicate head (cranial) and tail (caudal) orientation. (C) Representative H\&E images of EUS-FNAderived PDOs and PDOXs $(n=3)$. (D) Representative H\&E images of resection specimen-derived PDOs, corresponding PDOXs and primary tumor (PT), when available $(n=3)$. Scale bars: $300 \mu \mathrm{m}$ (main image), $60 \mu \mathrm{m}$ (inset). (E) Color-coded table of PDAC IHC subtypes in PT from FNA (when available) (patient ID number 42), or surgical resections (patient ID numbers 25, 54, and 61), PDO and corresponding PDOX. n.a., not available (cytology-negative FNAs); n.c., not classifiable (HNF1A/KRT81 double positive); PDO, patient-derived organoid; PDOX, PDO-derived xenografts.

Based on a recent study by the Cancer Genome Atlas Research Network, most PDACs can be classified into 2 major subtypes - classical/pancreatic progenitor subtype and basal-like/squamous (18-22). Notably, the squamous subtype displays a poorer prognosis compared with the classical/progenitor subtype (18-21).

Similar to previous studies, the subtyping was not entirely stable between the PT (when available), PDO, and PDOX (Figure 1E and Supplemental Figure 2). These findings demonstrate that pancreatic 
cancers as well as PDO from these tumors harbor a remarkable potential to switch between molecular subtypes, underscoring the inherent plasticity of these cells.

cfDNA in PDO supernatant is a valuable tool for detecting mutations shortly after biopsy. We next aimed to reduce the time required for the PDO-augmented diagnosis and molecular characterization of PDAC. In patients with insufficient amounts of biopsy material, which can be attributed to low input cellularity or limited tumor cell fraction, an enrichment of tumor cell signal might increase diagnostic depth. Additionally, to perform clinically meaningful and personalized drug testing, knowledge of the mutational profile of the PDO is required. Therefore, we hypothesized that from the very beginning, after biopsy in vitro, cfDNA released from the PDO cells could be used to detect potentially druggable mutations of the corresponding PDAC patient. At the same time, the expansion process of an individual PDO line for further functional analyses would not be disrupted and, hence, allow earlier functional testing. To this end, PDO-SNs were harvested starting from 72 hours after biopsy. As a proof of concept, the supernatant was subjected to digital droplet PCR (ddPCR) to detect $K R A S^{G 12 D}, K R A S^{G 12 V}$, and $K R A S^{G 12 R}$ mutations (Figure 2, A and B; and Supplemental Tables 3 and 4). In addition, we determined the mutant allele frequency (MAF) of indicated KRAS mutations in PDOs $(n=7)$ (Figure 2, B and C). We further compared the MAFs of KRAS mutations in the PT (FNA or Res.), PDO, and corresponding PDO-SN $(n=3)$ (Figure $2 \mathrm{~B})$. Notably, we observed almost identical MAFs of KRAS mutations in PDO-SN compared with the PT and PDO (Figure 2B). For example, the PT of patient ID number 42 had a mutant $K R A S^{G 12 V}$ allele frequency of $38 \%$, whereas the corresponding PDO and PDO-SN showed a MAF of $60 \%$ and 39\%, respectively (Figure 2B and Supplemental Table 4). We next compared macrodissected tissues from PDOXs to corresponding PDOs and PDO-SNs ( $n$ = 4) (Figure 2C and Supplemental Table 4). Again, the MAF of KRAS mutations was highly reproducible. For instance, patient ID number 34 showed a $96.0 \%$ MAF of $K R A S$ mutations in the PDO-SN versus $100 \%$ and $99.7 \%$ in PDO and PDOX, respectively (Figure 2C and Supplemental Table 4). Because the allele frequencies of mutant KRAS were remarkably stable in PTs (when available), PDOs, PDOXs, and PDO-SNs, we next sought to infer the mutational landscape of a given patient tumor by subjecting the PDO-SN to next-generation sequencing (Figure 2D and Supplemental Table 4). Mutations that were present in normal tissue controls (germline variants) have been excluded from the analyses. Those patients for whom we had no access to normal tissue, we dismissed mutations that have not been described previously in the Genome Aggregation Database (gnomAD), the Catalog of Somatic Mutations in Cancer (COSMIC), and ClinVar databases to exclude false-positive results. When we applied panel sequencing to PDO-SNs, we were able to obtain highly matching results from the PT (when available), PDO, PDO-SN, and PDOX (Figure 2D and Supplemental Table 4). To demonstrate feasibility, indicated PDO lines were subjected to drug testing with standard chemotherapeutics (Supplemental Figure 3). In line with previous reports $(4,9)$, we observed a pronounced heterogeneity in response toward chemotherapy (Supplemental Figure 3B).

\section{Discussion}

The pronounced inter- and intrapatient heterogeneity, distinct morphologic and molecular subtypes, and the fact that most mutations occur with a frequency of under $5 \%$ in pancreatic cancer (22) requires analyses of large patient cohorts for definition and characterization of clinically relevant pancreatic cancer subgroups. In the past, patients with advanced pancreatic cancer have not been studied to the same extent as resected patients owing to the lack of sufficient amount of tumor material. Additionally, the high proportion of stromal cells and low tumor cellularity in PDAC makes the genetic profiling of this disease more challenging. PDOs, however, possess a pure epithelial phenotype and, therefore, harbor a decreased signal-to-noise ratio when characterized, for example, by sequencing compared with the PT and thereby increasing sensitivity. Notably, mutations identified in PDOs have been demonstrated to harbor potential implications for the development of novel therapeutic approaches in the context of personalized medicine (4). In general, molecular subtyping based on gene expression profiling taught us that the complexity of pancreatic cancer can be reduced to allow meaningful patient stratification $(1,19,21,22)$. However, although the genotype of the PT and corresponding PDO as well as PDOX is conserved, the phenotype determined by IHC subtyping as well as gene expression profiling is not entirely stable. These results are in line with the bladder cancer study by Lee and colleagues, in which approximately two-thirds of the samples undergo significant phenotypic changes, indicating pronounced cellular plasticity and a potential limitation of this strategy in the PDO model systems (23).

Here, we mainly focus on PDOs from EUS-FNAs. In the near future, EUS-FNAs will become the main source of patient material to generate therapy-naive PDOs considering that most patients, even 
A

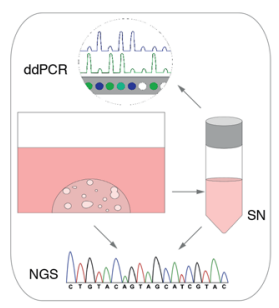

B

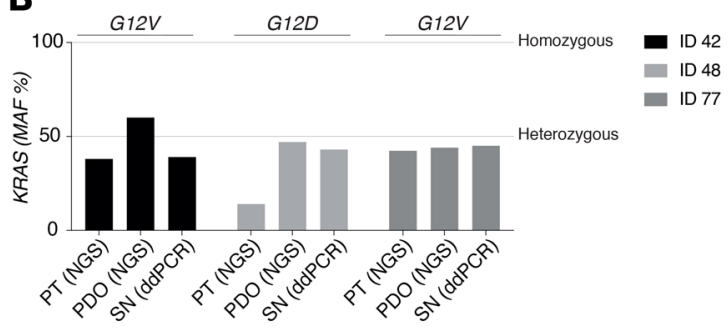

C
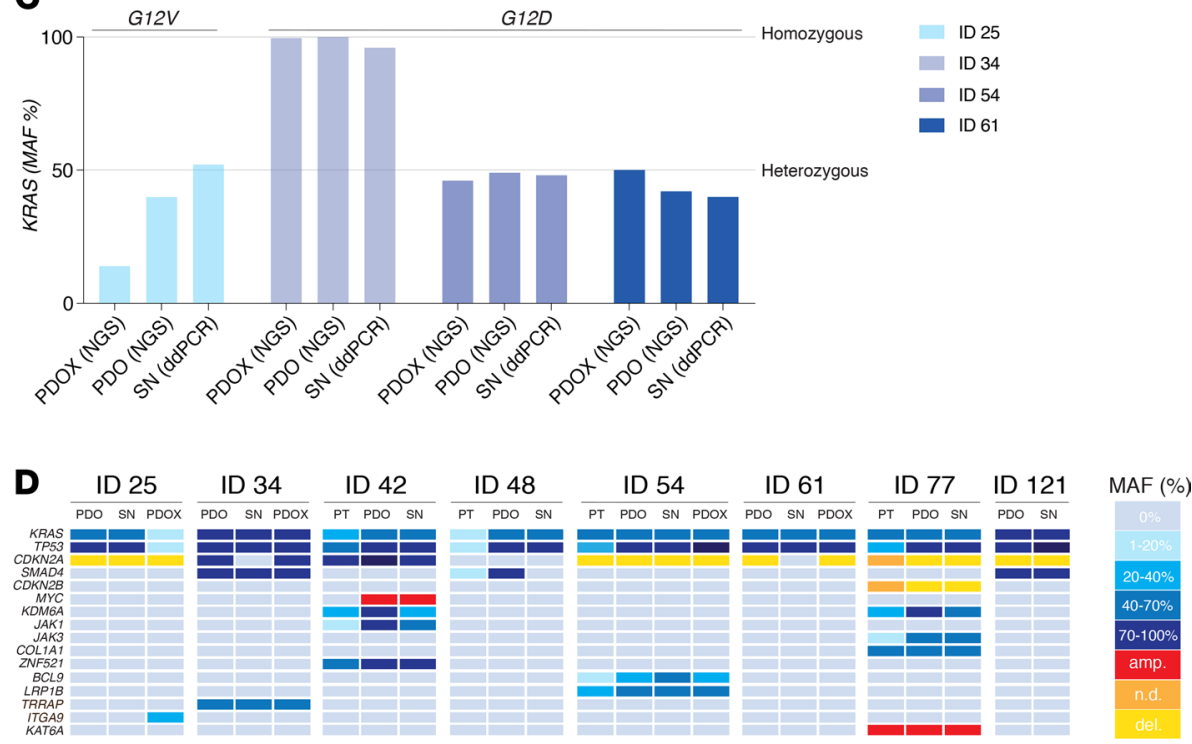

Figure 2. Detection of tumor-specific mutations in PDO-SN. (A) Experimental workflow to analyze PDO-SNs. (B) KRAS mutations detected in primary tumor samples ( $n=3$; patient ID numbers 42,48 , and 77), corresponding PDO (NGS), and PDO-SN (ddPCR). (C) KRAS mutations detected in PDOX ( $n=4$; patient ID numbers $25,34,54$, and 61 ), corresponding PDO (NCS), and PDO-SN (ddPCR). (D) Mutational profile of matched PT, PDO, and SN by NGS. amp., amplification; ddPCR, digital droplet PCR; del., deletion; MAF, mutant allele frequency; n.a., not available; n.d., not detectable; NGS, next-generation sequencing; PDO, patient-derived organoid; PDOX, PDO-derived xenografts; PT, primary tumor, SN, supernatant.

those eligible for surgery, will have to receive a biopsy before neoadjuvant therapy. In this study, we were especially interested in characterizing PDOs that have been generated from biopsies that were considered tumor negative based on cytological and/or histopathology findings. However, even biopsies with low tumor cellularity that allow establishing definitive diagnoses frequently harbor insufficient amounts of material required for further molecular profiling and functional testing. In these cases, PDOs generated from EUS-FNAs to expand material for subsequent sequencing and functional downstream applications are particularly valuable since up to $40 \%$ of pancreatic cancers have mutations that are potentially druggable (20). Similar to previous studies $(4,15)$, our PDOs match the genetic landscape of the PT. Notably, by subjecting cfDNA from PDO-SNs to next-generation sequencing (NGS), we were able to detect the mutational profile of the PT without limiting the expansion process of a given PDO line. This accelerated molecular characterization potentially allows genotype-guided drug testing within a substantially reduced turnaround time after biopsy, which might be an advantage to implement clinical workflows, especially in stage IV disease. In addition, in the field of PDOs it has become apparent that normal, nontransformed cells are able to grow under standard organoid culture conditions (24). However, this limitation can be reduced, not completely prevented, by applying more stringent, growth factor-reduced media conditions (24). Therefore, an early assessment of cfDNA in the conditioned media might be useful to confirm the presence of oncogenic mutations. Additionally, analyzing defined mutations by ddPCR could be a valuable and cost-effective quality measurement. Moreover, assessing cfDNA in the PDO supernatant might be able to provide insights on cfDNA dynamics during therapy to inform biomarker development to monitor treatment responses. 
Overall, our findings suggest that qualitative assessment of cfDNA is an advancement in PDO technology. Generating PDOs from a single FNA might increase diagnostic accuracy, allowing molecular profiling and subtyping as well as functional drug testing. This is particularly relevant in patients for whom biopsy samples were rejected from all genetic testing owing to insufficient tumor quantity. Our integrated approaches, in combination with a rapid turnaround time from biopsy to functional result, might facilitate the integration of PDO technology into clinical trials and, eventually, impact treatment decisions made by the oncologist.

\section{Methods}

Patient cohort. Samples were received from EUS-FNA in the endoscopy suite at Klinikum rechts der Isar, Technical University Munich, or from resection specimens from the Surgical Departments at the Klinikum rechts der Isar or University Hospital Carl Gustav Carus, Technical University Dresden (1 sample). EUSFNA was performed for initial diagnosis. A second pass was performed with a 19-, 20-, or 22-gauge FNA needle for the 3D organoid biobank (Supplemental Table 1).

PDO generation from EUS-guided FNA and resection specimens. The isolation process is similar to what has been described previously with some modifications $(15,25)$. Organoid isolation was started within 15 minutes after receiving the sample. After washing with washing or splitting media (Advanced DMEM/F12 with 1X GlutaMax, $10 \mathrm{mM} \mathrm{HEPES,} \mathrm{and} 100 \mu \mathrm{g} / \mathrm{mL}$ Primocin) and a centrifugation step ( 5 minutes, $4^{\circ} \mathrm{C}$, and $200 \mathrm{~g}$ ), the supernatant was discarded and the sample was dissected into small pieces and incubated with RBC lysis buffer (ACK lysis buffer, Life Technologies) for 10-15 minutes at room temperature. Then, the sample was digested. Specifically, FNA samples were dissociated by enzymatic digestion for 5 to 10 minutes with $500 \mu \mathrm{L}$ to $1 \mathrm{~mL}$ of TrypLE (Life Technologies) at $37^{\circ} \mathrm{C}$. Resection specimens were subjected to a 1-2 hours of collagenase digest (collagenase type II, Life Technologies) at $5 \mathrm{mg} / \mathrm{mL}$. The sample was washed further (with washing media) and centrifuged. Next, the pellet was mixed with Matrigel, and $50 \mu \mathrm{L}$ of the Matrigel and cell mixture was plated in each well of a prewarmed 24-well plate. After incubation of the plate for 20 minutes, $500 \mu \mathrm{L}$ of warm feeding media was added to the well. For the biobank at our institution (Klinikum rechts der Isar, Technical University of Munich), the PDOs were cultured in 2 distinct types of media conditions with equal performance after the biopsy cell pellet had been divided into 2 equal parts. The normal feeding media (NFM) contained the following components: AdDMEM/F12 medium supplemented with HEPES (10 mM, Life Technologies), GlutaMax (1X, Life Technologies), B27 (1X, Life Technologies), Primocin (100 $\mu \mathrm{g} / \mathrm{mL}$, InvivoGen), N-acetyl-L-cysteine (1.25 mM, MilliporeSigma), recombinant human Wnt3a protein (100 $\mathrm{ng} / \mathrm{mL}, \mathrm{R} \& \mathrm{D}$ Systems) or Wnt3a-conditioned medium (50\%), RSPO1-conditioned medium (10\%) or recombinant human R-Spondin 1 protein (500 ng/mL), mNoggin (100 ng/mL, Preprotech), EGF (50 ng/mL, Life Technologies), Gastrin (10 nM, MilliporeSigma), FGF10 (100 ng/mL, Preprotech), Nicotinamide (10 mM, MilliporeSigma), Y-27632 (10 $\mu \mathrm{M}$, MilliporeSigma), and A83- 01 (0.5 $\mu \mathrm{M}$, Tocris).

The modified NFM media contained the following components: DMEM/F12 or AdDMEM/F12 supplemented with Pen/strep (1\%), Primocin (100 $\mathrm{g} / \mathrm{mL}$, InvivoGen), Nu-Serum IV (5\%, Corning), D-Glucose (5 mg/mL, MilliporeSigma), Nicotinamide (10 mM, MilliporeSigma), Soybean Trypsin Inhibitor (optional) (0.1 mg/mL, Life Technologies), ITS+premix (0.5\%, Life Technologies), recombinant human Wnt3a protein (50 or $100 \mathrm{ng} / \mathrm{mL}, \mathrm{R} \& \mathrm{D}$ Systems) or Wnt3a-conditioned medium (50\%), RSPO1-conditioned medium (10\%) or recombinant human R-Spondin 1 protein $(500 \mathrm{ng} / \mathrm{mL}$, R\&D Systems), 3,3,5-Triiodo-L-thyronine (5 $\mathrm{nM}$, MilliporeSigma), Dexamethasone $(1 \mu \mathrm{M})$, Choleratoxin $(100 \mathrm{ng} / \mathrm{mL})$, Epidermal growth factor (EGF, $20 \mathrm{ng} / \mathrm{mL}$ or $50 \mathrm{ng} / \mathrm{mL}$, Life Technologies), Bovine Pituitary Extract (25 $\mu \mathrm{g} / \mathrm{mL}$, Life Technologies), and A83-01 (0.5 $\mu \mathrm{M}$, Tocris).

PDO passaging. After aspiration of feeding media, $500 \mu \mathrm{L}$ of ice-cold splitting media (Advanced DMEM/F12 with 1X GlutaMax, $10 \mathrm{mM}$ HEPES, and $100 \mu \mathrm{g} / \mathrm{mL}$ Primocin) was added to the well, and the Matrigel and organoid mixture was resuspended with the splitting media, transferred into a $15-\mathrm{mL}$ falcon tube, and centrifuged at $200 \mathrm{~g}$ for 5 minutes at $4^{\circ} \mathrm{C}$. After centrifugation, the supernatant was removed until $1 \mathrm{~mL}$ of the splitting media remained. The organoids were broken further using the fire-polished pipettes. Then, the falcon tube was filled with the cold splitting media and centrifuged at $200 \mathrm{~g}$ for $5 \mathrm{~min}$ utes at $4^{\circ} \mathrm{C}$. Next, the supernatant was aspirated, the pellet was resuspended with the Matrigel, and $50 \mu \mathrm{L}$ of the organoid mixture and Matrigel was seeded in each well of a 24-well plate. After solidification of the Matrigel, $500 \mu \mathrm{L}$ of warm feeding media was added in each well. 
PDO freezing. The organoids were treated exactly as previously described in the splitting step, including the second wash step when there was a pellet. Then, the pellet was resuspended in $1 \mathrm{~mL}$ of Recovery Cell Culture Freezing Medium (Life Technologies) per each cryovial.

PDOXs. Female athymic nude mice (NU[NCr]-Foxn $1^{n u}$ ) were ordered from Charles River at 8-10 weeks of age. After mechanic and enzymatic digestion of organoids into single cells, 300,000-500,000 cells were mixed with $50 \mu \mathrm{L}$ of Matrigel and PBS (1:1 ratio). Subsequently, the previously mentioned cell mixture was injected orthotopically into athymic nude mice. Animals were imaged by PET and MRI 50 days after transplantation. After euthanasia of mice, the pancreas was harvested for histological analysis (Supplemental Table 1).

In vivo analysis of tumors by PET and MRI imaging. The animals were anesthetized with isoflurane $\left(1.5 \% \mathrm{O}_{2}, 2 \%\right.$ Isofluran) and monitored under constantly warm conditions. The PET and MRI imaging experiments were performed at 50 days after transplantation. The animals were imaged consecutively using the Siemens Inveon microPET/CT (Siemens Healthcare) and 7T MRI (MR901 Agilent, GE, AVANCE III HD electronics, Bruker 31-mm inner diameter volume coil, RAPID Biomedical). The abdomen was immobilized using a rapidly setting mold (26) using the MR-compatible material alginate (Creato Alginat Abformmasse, Zitzmann Zentrale) to prevent deformation during the transport between imaging modalities and to allow for precise fusion of PET and MRI. A total of $10-15 \mathrm{MBq}{ }^{18}$ fluorodeoxyglucose was injected intravenously after a CT scan, and dynamic PET data were acquired in list mode for 50 minutes. All PET measurements were corrected for physical decay, dead time, and nonuniformity of microPET response, and the images were reconstructed using the $3 \mathrm{D}$ ordered subset expectation maximization algorithm. T2-weighted (T2-w) MRI was performed after PET/CT acquisition using a Rapid Acquisition with Relaxation Enhancement (RARE) sequence (echo time [TE]/repetition time [TR]/flip angle $=42.7 \mathrm{~ms} / 20,000 \mathrm{~ms} / 90^{\circ}$ ). For anatomical T2-w imaging, mice were kept in the prone position after the PET imaging to ensure coregistration and scanned at the 7T MRI system with the H-1 volume resonator coil. A coronal multislice T2-w RARE sequence (resolution $0.2 \times 0.2 \times 0.4 \mathrm{~mm}^{3}$, TE $=45.5$ $\mathrm{ms}, \mathrm{TR}>5 \mathrm{~s}$ ) was applied for tumor detection.

Histology. For histology analyses, patient ID numbers 34, 42, and 66 FNA samples were embedded into paraffin. Primary FNA material of diagnostic quality was only available in 1 of the 3 cases (patient ID number 42) (Supplemental Table 1). PTs of patient ID numbers 25, 54, and 61 were available after surgical resection. Tissue blocks from surgical resections and cell blocks from FNAs were routinely processed. PDOs were fixed in $4 \%$ formaldehyde for 1 hour and PDOXs for at least 48 hours, dehydrated, and embed$\mathrm{ded}$, and serial $2-\mu \mathrm{m}$-thin paraffin sections were prepared. H\&E staining was performed on deparaffinized sections with Eosin and Mayer's hematoxylin according to a standard protocol for routine diagnostics.

Immunohistochemistry of the cell blocks from the FNA, PTs, and PDOs was performed on a BenchMark XT automated stainer (Ventana) with HNF1A (Santa Cruz 8986) and KRT81 (Santa Cruz 100929) antibody (Supplemental Table 5) using the ultraVIEW DAB Detection Kit (Ventana). After deparaffinization, the slides were incubated with the primary antibodies diluted in antibody diluent (1:200 dilution, 1:250 dilution for HNF1A and KRT81, respectively) (Supplemental Table 5) at $37^{\circ} \mathrm{C}$. Antibody binding was detected using $\mathrm{DAB}$ as chromogen and counterstained with hematoxylin for 8 minutes with subsequent bluing. Slides were then dehydrated manually with alcohol at increasing concentration, cleared with xylene, and coverslipped.

Immunohistochemistry of PDOXs was performed using a Bond RXm system (Leica, all reagents were from Leica) with primary antibodies against HNF1A (Santa Cruz 8986) and KRT81 (Santa Cruz 100929). Slides were deparaffinized, pretreated with Epitope retrieval solution 1 (corresponding to citrate buffer, pH 6). The primary antibodies were diluted (1:50 dilution, 1:200 dilution for HNF1A and KRT81, respectively) (Supplemental Table 5) and applied for 15 minutes. Antibody binding was detected with a polymer refine detection kit (without postprimary reagent for HNF1A) and visualized with $\mathrm{DAB}$ as a dark-brown precipitate. Counterstaining was performed with hematoxylin. Slides were then dehydrated and coverslipped. For KTR81 staining, a Vector M.O.M. Kit was used to specifically reduce the background staining. A positive control was included in each run.

The stained slides were evaluated by certified pathologists who were blinded to the clinical information. The percentage and the color intensity of the positive tumor cells regarding different antibodies were documented. PDACs were subtyped according to previously published criteria (17) (Supplemental Table 2). The stained slides were scanned with an automated slide scanner (Leica Biosystems, AT-2), and the Aperio ImageScope software (version 12.3, Leica Biosystems) was used to take representative images. 
Preparation of cfDNA from PDO-SN for ddPCR and next-generation sequencing. To perform ddPCR on PDOSN for detection and quantification of KRAS mutations G12D, G12V, and G12R, the SN was harvested and DNA was extracted from $1 \mathrm{~mL}$ of SNs with the QIAamp UltraSens Virus Kit (QIAGEN) and quantified using a Qubit 2.0 fluorometer (Thermo Fisher Scientific). The median concentration of DNA in the supernatant was $25 \mathrm{ng} / \mathrm{mL}$ (IQR 20-39 ng/mL). For KRAS mutation detection, one-fifth of the extracted DNA was used as input for ddPCR on a QX200 ddPCR system with automated droplet generation (Bio$\mathrm{Rad}$ ). For next generation sequencing of the PDO-SN, 1.5-5 mL of cell culture supernatant was used. The DNA extraction was performed using the Maxwell RSC ccfDNA Plasma Kit (Promega) according to the recommendation of the manufacturer for isolating cfDNA. Samples with DNA concentrations below $1 \mathrm{ng} /$ $\mu \mathrm{L}$ were then concentrated using Vivacon-500 columns (size: $30,000 \mathrm{kD}$, Sartorius).

ddPCR. The SN of PDOs was harvested and submitted to the Institute of Clinical Chemistry and Pathobiochemistry at Klinikum rechts der Isar for detection and quantification of KRAS mutations G12D, G12V, and G12R using ddPCR. DNA was extracted from $1 \mathrm{~mL}$ of supernatant samples with the QIAamp UltraSens Virus Kit (QIAGEN) and quantified using a Qubit 2.0 fluorometer (Thermo Fisher Scientific). Samples were prepared according to the manufacturer's protocol and eluted in a volume of $60 \mu \mathrm{L}$. The final concentration was then calculated based on the DNA amount eluted in $60 \mu \mathrm{L}$ and the volume of the supernatant $(1 \mathrm{~mL})$. The median concentration of DNA in the supernatant was $25 \mathrm{ng} / \mathrm{mL}$ (IQR $20-39 \mathrm{ng} / \mathrm{mL}$ ). For KRAS mutation detection, one-fifth of the extracted DNA was used as input for ddPCR on a QX200 ddPCR system with automated droplet generation (Bio-Rad). Reactions were carried out in ddPCR 96-well plates (catalog 12001925, Bio-Rad). Each well contained $10.5 \mu \mathrm{L}$ of ddPCR Supermix for Probes (no dUTP; Bio-Rad), $1.05 \mu \mathrm{L}$ of target-specific primers (900 nmol/L), $1.05 \mu \mathrm{L}$ of target-specific probe (250 nmol/L), $1.05 \mu \mathrm{L}$ of $\mathrm{MseI}$ restriction enzyme (New England Biolabs), $1.05 \mu \mathrm{L}$ of water, and $6.3 \mu \mathrm{L}$ of sample DNA for a total volume of $21 \mu \mathrm{L}$. Target assays were G12D (dHsaCP2000001, dHsaCP2000002, Bio-Rad), G12V (dHsaCP2000005, dHsaCP2000006, Bio-Rad), and G12R (dHsaCP2000009, dHsaCP20000010, Bio-Rad). Positive controls (gBlocks, synthetic DNA with the amplicon sequence and KRAS mutation or KRAS WT), NTC (purified, nuclease-free water), and negative controls (10 ng of genomic DNA from peripheral blood leukocytes of healthy subjects) were included on every plate for each assay. Plates were sealed, spun down, and loaded into the droplet generator. Immediately after droplet generation, 96-well plates containing droplet-partitioned samples were sealed, and PCR was carried out on a C1000 Touch Thermal Cycler (Bio-Rad) using the following cycling protocol: enzyme activation at $95^{\circ} \mathrm{C}$ for 10 minutes followed by 40 cycles of $94^{\circ} \mathrm{C}$ for 30 seconds (for denaturation) and $55^{\circ} \mathrm{C}$ for 60 seconds (for annealing/extension), followed by a final 10 -minute incubation at $98^{\circ} \mathrm{C}$ (for enzyme deactivation). Ramp rate was $2^{\circ} \mathrm{C}$ per second. Plates were then kept at $4^{\circ} \mathrm{C}$. All samples were measured in duplicates (2 wells). Plates were read on a QX200 droplet reader (Bio-Rad). Raw droplet fluorescence intensity values were exported from QuantaSoft droplet reader software v1.7.4 (Bio-Rad). Custom scripts were used to import the intensity values into R (version 3.4.4; http://www.r-project.org) and to quantify concentrations of KRAS mutant and KRAS WT DNA. Target concentrations (c) were calculated for each well from the number of positive droplets $(\mathrm{Np})$ and negative droplets $(\mathrm{Nn})$ and the average droplet volume $(\mathrm{V}=0.85 \mathrm{~nL})$ based on Poisson distribution statistics using the formula $\mathrm{c}=(\ln [\mathrm{Np}+\mathrm{Nn}]-\ln [\mathrm{Nn}]) / \mathrm{V}$, where $\ln$ is the natural logarithm. The number of partitions (droplets) per reaction was 16,855 on average (range 11,837-21,738, SD 2743). With a droplet volume of $0.85 \mathrm{~nL}$ reported by Bio-Rad, the effective reaction size (total volume of partitions measured) was $14.3 \mu \mathrm{L}$ on average (range 10.1-18.5 $\mu \mathrm{L}, \mathrm{SD} 2.3 \mu \mathrm{L}$ ).

Next-generation library preparation, DNA sequencing, and data analysis. Cell pellets from FNA were routinely processed using centrifugation-based cell enrichment in Histogel (Thermo Scientific) and embedded in Paraffin. A total of 5- $\mu \mathrm{m}$-thin sections were prepared using a rotary microtome (HM355), and slides were mounted on PEN membrane-coated slides (Leica Biosystems) or standard slides (StarFrost, Engelbrecht). Laser capture microdissection (LCM) was performed by annotation and dissection of tumor cells from 3 sections in 1 patient (see below). For DNA extraction from FFPE specimens, 8- $\mu$ m-thick sections of FFPE tumor specimens were deparaffinized and digested with Proteinase K overnight. Automated extraction of nucleic acids was performed using the Maxwell 16 RSC extraction system (Promega). DNA concentration was fluorimetrically determined using the Qubit 3.0 system (Thermo Fisher Scientific), and DNA quality was additionally tested by a qPCR assay (RNaseP assay, Thermo Fisher Scientific) as previously described (27). For the one cytological sample (patient ID number 42) in which only cytological material was available, laser microdissection was performed using a Leica LMD6 system according to the manufacturer's instructions. 
To enrich for tumor cells, the FNA specimen was spun down and embedded as cell pellet using HistoGel (Thermo Fisher Scientific). DNA from the laser-microdissected sample was isolated using the FFPE direct kit (Thermo Fisher Scientific). Briefly, $10 \mu \mathrm{L}$ of transfer solution was directly added to the laser-microdissected tissue areas for cell lysis and mixed by pipetting. After the addition of $21 \mu \mathrm{L}$ of direct reagent, the mixture was incubated at $65^{\circ} \mathrm{C}$ in a thermocycler for 15 minutes followed by up to 30 minutes at $20^{\circ} \mathrm{C}$. The DNA was quantified by Qubit measurement. The DNA concentration after LCM was $0.191 \mathrm{ng} / \mu \mathrm{L}$ (patient ID number 42). For each of the 4 libraries, $6 \mathrm{~mL}$ of the extraction were used for amplification.

For panel sequencing of the conditioned media, we used the cell culture supernatant for DNA extraction using the Maxwell RSC ccfDNA Plasma Kit (Promega) according to the recommendation of the manufacturer for isolating cfDNA. A total of $1.5-5 \mathrm{~mL}$ of cell culture supernatant was incubated after adding 1 volume of binding buffer and one-tenth volume of Proteinase $\mathrm{K}$ for 15 minutes at $56^{\circ} \mathrm{C}$ in a water bath. The beads from the second well of the Maxwell cartridge were rebuffered and/or washed with isopropanol. After removal of the isopropanol the beads were resuspended in $1 \mathrm{~mL}$ of binding buffer and then added to the Proteinase $\mathrm{K}$ digested cell culture supernatant and incubated for 45 minutes on a roller shaker at room temperature. After centrifugation of the mixture for 2 minutes at $200 \mathrm{~g}$, the supernatant was removed, leaving approximately $1 \mathrm{~mL}$ behind. The beads with bound DNA were resuspended and pipetted back to the second well of the cartridge. DNA isolation was then performed on the Maxwell RSC16 system using the Maxwell large volume cfDNA custom protocol. Samples with DNA concentrations below $1 \mathrm{ng} / \mu \mathrm{L}$ were then concentrated using Vivacon 500 spin columns (size: $30,000 \mathrm{kD}$, Sartorius). The complete DNA was added to the column and centrifuged for about 10 minutes at $11,000 \mathrm{~g}$. The remaining liquid containing the concentrated DNA was then collected by inverting the column to a new centrifuge tube and centrifuging for about 30 seconds at $11,000 \mathrm{~g}$.

Libraries were prepared by applying the AmpliSeq Comprehensive Cancer Panel (AmpliSeq CCP, Thermo Fisher Scientific) consisting of 4 primer pools for amplification of 15,992 amplicons covering almost the complete exonic regions of 409 cancer-related genes. Semiconductor sequencing was performed as previously described $(27,28)$ on an Ion S5XL sequencing system using the Ion Chef 540 sequencing chemistry and a 540 Chip. Raw sequencing data were processed in the Torrent Suite Software (version 5.8.0) and aligned against the human genome (version hg19) using the TMAP algorithm. The built-in variantCaller (version 5.8.0.19) and Coverage Analysis (version 5.8.0.8) reports were used for variant calling and to generate the coverage data. Variant annotation was performed by a custom-built variant annotation pipeline using ANNOVAR (29). Variants were visualized using the Integrative Genomics Viewer Browser (http://www.broadinstitute.org/igv/) and were checked for germline or somatic origin using the COSMIC (30), dbSNP, and the Exome Aggregation Consortium (31) databases. Identification of copy number variations (amplifications and deletions) was performed for each sample and amplicons using the coverage data summary generated by the Torrent Suite software using a 4-step algorithm as previously described (27, 32).

For whole-exome sequencing, the DNA isolation from PDOs and primary samples (normal and tumor tissues) was conducted using AllPrep DNA/RNA/miRNA Universal Kit (QIAGEN), according to the manufacturer's instructions. DNA isolation from blood was performed using QIAamp DNA Mini Kit (QIAGEN) according to the manufacturer's instructions.

Libraries for whole-exome sequencing were prepared using the Agilent SureSelect Human All Exon v6, according to the manufacturer's instructions, and sequenced on an Illumina HiSeq 4000 sequencer (ID 48, 54, 61). For patient ID numbers 25, 34, 42, and 121, the Agilent SureSelect Low Input Human All Exon v6 library preparation kit was used, and samples were sequenced on an Illumina NovaSeq 6000. All samples were sequenced to a median coverage of approximately $\times 100$. The GATK Best Practice suggestions were followed for mutation calling. After read trimming using Trimmomatic 0.38 (LEADING:25 TRAILING:25 MINLEN:50), BWA-MEM 0.7.17 was used to map reads to the reference genome (GRCh38.p12). Picard 2.18.26 and GATK 4.1.0.0 were used for postprocessing (CleanSam, MarkDuplicates, BaseRecalibrator) using default settings. Somatic mutations were called using MuTect2 v4.1.0.0. Mutations with at least 2 reads supporting the alternate allele and an overall base coverage of at least 10 in the tumor, and where available in the germline sample, were required. The gnomAD (33) was used to evaluate putative germline variants for samples in which germline information was not available. Single-nucleotide variants and indels less than or equal to $10 \mathrm{bp}$ were annotated using SnpEff 4.3t, based on ENSEMBL 92. Copywriter 2.6.1.2 was used for the detection of copy number variations.

Pharmacotyping of PDOs. One thousand cells per well in a total volume of $80 \mu \mathrm{L}$ of medium and 10 $\mu \mathrm{L}$ of Matrigel were seeded in each well of a white 96-well plate (Corning, cat. no. 3610), which was 
coated with a $30-\mu \mathrm{L}$ mixture of Matrigel and PBS (1:3 or 1:4 ratio). After 24 hours, a 7-point dilution of compounds was added to the corresponding wells. The therapeutic reagents gemcitabine, 5-fluorouracil, oxaliplatin, cisplatin, carboplatin, irinotecan, and paclitaxel were provided by the Pharmacy at Klinikum rechts der Isar, Technical University of Munich. Bortezomib was purchased from LC-Laboratories. The drugs were dissolved according to the manufacturer's instructions. For in vitro treatment of PDOS, the compounds were dissolved in DMSO or water. Each therapeutic compound was tested between 2-10 times. Three to 5 days after treatment, based on the growth of different PDOs, the cell viability was measured using CellTiter-Glo 3D Cell Viability Assay according to the manufacturer's instructions (Promega). The CellTiter-Glo 3D Cell Viability Assay was thawed at room temperature, and $100 \mu \mathrm{L}$ of the solution was added to each well of the 96-well-plate containing organoids, Matrigel, and medium. Then, the plate was placed on a rotator for 10-15 minutes. Next, the plate was measured using a Fluostar Optima (BMG Labtech) on the luminescence setting. The analysis was performed using GraphPad Prism 7.

Study approval. All patients were recruited, enrolled, and signed informed consent forms based on the IRB project number 207/15 and 1946/07 of the Technical University Munich and EK451122014 in Dresden. All animal experiments and care were in accordance with the guidelines of institutional committees and approved by the local authority, Regierung von Oberbayern, project number 55.2-1-54-2532.0-54-2016.

\section{Author contributions}

$\mathrm{ZD}$ and $\mathrm{MR}$ conceived and designed the study. ZD, FO, AH, MRO, DS, and MR generated the organoid lines. ZD performed organoid experiments. ZD and MW performed drug screens. SL, TE, RÖ, KP, KF, AP, APGS, RR, and CLM provided experimental support. ZD and MR performed animal experiments. ZD, SL, and CLM created the figures. AH and LL performed study patient enrollment. TW, MM, GOC, and HF performed surgery and provided the surgical specimens for the organoid biobank. MT, GVF, MA, HA, PK, CS, JS, and DS performed endoscopic ultrasound and provided the FNA samples for the organoid biobank. HY, KS, AM, and WW performed histology staining, subtyping, and data analysis. SL, RÖ, TE, and NP performed the NGS, WXS, and data analysis. CW performed ddPCR and ddPCR data analysis. KS and GT performed PET/MRI imaging. IH, GK, and RB performed PET/MRI imaging analysis. ZD and MR wrote the manuscript. RR, RMS, and GS revised the manuscript. MR supervised the overall conduct of the study. All authors reviewed and approved the manuscript.

\section{Acknowledgments}

We dedicate this work to the memory of our patients. We thank all patients and their families. This work was funded by the AGA Moti L. \& Kamla Rustgi International Travel Award to ZD. MR was supported by the German Cancer Aid (Max Eder Program, Deutsche Krebshilfe 111273, MR) and the German Research Foundation (DFG, SFB1321 Modeling and Targeting Pancreatic Cancer, Projects S01 and TP12, project ID 329628492). JTS is supported by the DKTK, the German Cancer Aid (grant no. 70112505; PIPAC consortium), and the DFG through grant SI1549/3-1 (Clinical Research Unit KFO337) and SFB824 (project C4). GS was supported by German Research Foundation (DFG SFB 1321/S01 and SCHN 959/3-1) and the DKTK Joint funding program. HYY, KS, and WW were supported by the German Research Foundation (DFG SFB824 and SFB1321). RB was supported by the German Research Foundation (DFG SFB824). The authors acknowledge Reiner Dunkl, Jana Grosz, Yukio Iwamoto, Charlotte Spitzner, Aylin Aydemir, and Lukas Zottl for technical support; Olga Josefina Seelbach and Marion Mielke for IHC staining; Elisabeth Graf and Tim Strom for whole-exome sequencing at the Institute of Human Genetics, Helmholtz Zentrum Munich and Thomas Wochnig for next-generation sequencing, and Julia Horstmann and Jens-Peter Zimmermann for study assistance. We would also like to thank Sybille Reder and Markus Mittelhäuser for their technical assistance for PET imaging. We are grateful to Anil K. Rustgi (Herbert Irving Comprehensive Cancer Center, Columbia University) for his valuable input on the manuscript.

Address correspondence to: Maximilian Reichert, Trogerstrasse 32, Building 543, Room 1.04, 81675 Munich, Germany. Phone: 49.89.4140-9454; Email: maximilian.reichert@tum.de. 
1. Aguirre AJ, et al. Real-time genomic characterization of advanced pancreatic cancer to enable precision medicine. Cancer Discov 2018;8(9):1096-1111.

2. Siegel RL, Miller KD, Jemal A. Cancer statistics, 2017. CA Cancer J Clin. 2017;67(1):7-30.

3. Rahib L, Smith BD, Aizenberg R, Rosenzweig AB, Fleshman JM, Matrisian LM. Projecting cancer incidence and deaths to 2030: the unexpected burden of thyroid, liver, and pancreas cancers in the United States. Cancer Res. 2014;74(11):2913-2921.

4. Tiriac H, et al. Organoid profiling identifies common responders to chemotherapy in pancreatic cancer. Cancer Discov. 2018;8(9):1112-1129.

5. Huang L, et al. Ductal pancreatic cancer modeling and drug screening using human pluripotent stem cell- and patient-derived tumor organoids. Nat Med. 2015;21(11):1364-1371.

6. van de Wetering M, et al. Prospective derivation of a living organoid biobank of colorectal cancer patients. Cell. 2015;161(4):933-945.

7. Nanki K, et al. Divergent routes toward Wnt and R-spondin niche independency during human gastric carcinogenesis. Cell. 2018;174(4):856-869.e17.

8. Vlachogiannis G, et al. Patient-derived organoids model treatment response of metastatic gastrointestinal cancers. Science. 2018;359(6378):920-926.

9. Driehuis E, et al. Pancreatic cancer organoids recapitulate disease and allow personalized drug screening. Proc Natl Acad Sci U S A. 2019;null:201911273.

10. Sachs N, et al. A living biobank of breast cancer organoids captures disease heterogeneity. Cell. 2018;172(1-2):373-386.e10.

11. Tiriac H, et al. Successful creation of pancreatic cancer organoids by means of EUS-guided fine-needle biopsy sampling for personalized cancer treatment. Gastrointest Endosc. 2018;87(6):1474-1480.

12. Renz BW, et al. Cholinergic signaling via muscarinic receptors directly and indirectly suppresses pancreatic tumorigenesis and cancer stemness. Cancer Discov. 2018;8(11):1458-1473.

13. Renz BW, et al. $\beta 2$ adrenergic-neurotrophin feedforward loop promotes pancreatic cancer. Cancer Cell. 2018;33(1):75-90.e7.

14. Ruess DA, et al. Mutant KRAS-driven cancers depend on PTPN11/SHP2 phosphatase. Nat Med. 2018;24(7):954-960.

15. Boj SF, et al. Organoid models of human and mouse ductal pancreatic cancer. Cell. 2015;160(1-2):324-338.

16. Noll EM, et al. CYP3A5 mediates basal and acquired therapy resistance in different subtypes of pancreatic ductal adenocarcinoma. Nat Med. 2016;22(3):278-287.

17. Muckenhuber A, et al. Pancreatic ductal adenocarcinoma subtyping using the biomarkers hepatocyte nuclear factor-1A and cytokeratin-81 correlates with outcome and treatment response. Clin Cancer Res. 2018;24(2):351-359.

18. Collisson EA, et al. Subtypes of pancreatic ductal adenocarcinoma and their differing responses to therapy. Nat Med. 2011;17(4):500-503.

19. Moffitt RA, et al. Virtual microdissection identifies distinct tumor- and stroma-specific subtypes of pancreatic ductal adenocarcinoma. Nat Genet. 2015;47(10):1168-1178

20. Cancer Genome Atlas Research Network. Integrated Genomic Characterization of Pancreatic Ductal Adenocarcinoma. Cancer Cell. 2017;32(2):185-203.e13.

21. Chan-Seng-Yue M, et al. Transcription phenotypes of pancreatic cancer are driven by genomic events during tumor evolution. Nat Genet. 2020;52(2):231-240.

22. Waddell N, et al. Whole genomes redefine the mutational landscape of pancreatic cancer. Nature. 2015;518(7540):495-501.

23. Lee SH, et al. Tumor evolution and drug response in patient-derived organoid models of bladder cancer. Cell. 2018;173(2):515-528.e17.

24. Seino T, et al. Human pancreatic tumor organoids reveal loss of stem cell niche factor dependence during disease progression. Cell Stem Cell. 2018;22(3):454-467.e6.

25. Moreira L, Bakir B, Chatterji P, Dantes Z, Reichert M, Rustgi AK. Pancreas 3D organoids: current and future aspects as a research platform for personalized medicine in pancreatic cancer. Cell Mol Gastroenterol Hepatol. 2018;5(3):289-298.

26. Cho $\mathrm{H}$, et al. Noninvasive multimodality imaging of the tumor microenvironment: registered dynamic magnetic resonance imaging and positron emission tomography studies of a preclinical tumor model of tumor hypoxia. Neoplasia. 2009;11(3):247-259

27. Endris V, et al. Molecular diagnostic profiling of lung cancer specimens with a semiconductor-based massive parallel sequencing approach: feasibility, costs, and performance compared with conventional sequencing. J Mol Diagn. 2013;15(6):765-775.

28. Pfarr N, et al. Mutational profiles of Brenner tumors show distinctive features uncoupling urothelial carcinomas and ovarian carcinoma with transitional cell histology. Genes Chromosomes Cancer. 2017;56(10):758-766.

29. Wang K, Li M, Hakonarson H. ANNOVAR: functional annotation of genetic variants from high-throughput sequencing data Nucleic Acids Res. 2010;38(16):e164.

30. Forbes SA, et al. COSMIC: exploring the world's knowledge of somatic mutations in human cancer. Nucleic Acids Res. 2015;43(Database issue):D805-D811.

31. Lek M, et al. Analysis of protein-coding genetic variation in 60,706 humans. Nature. 2016;536(7616):285-291

32. Pfarr N, et al. Targeted next-generation sequencing enables reliable detection of HER2 (ERBB2) status in breast cancer and provides ancillary information of clinical relevance. Genes Chromosomes Cancer. 2017;56(4):255-265.

33. Karczewski KJ. The mutational constraint spectrum quantified from variation in 141,456 humans [preprints]. https://www. biorxiv.org/content/10.1101/531210v2?versioned=true. Posted on bioRxiv April 8, 2020. 